\title{
Safety of formoterol in asthma clinical trials: an update
}

\author{
Malcolm R. Sears ${ }^{1}$ and Finn Radner ${ }^{2}$
}

\section{Affiliations:}

${ }^{1}$ Firestone Institute for Respiratory Health, St Joseph's Healthcare and McMaster University, Hamilton, ON, Canada.

${ }^{2}$ AstraZeneca R\&D, Mölndal, Sweden.

Correspondence: M.R. Sears, Firestone Institute for Respiratory Health, St Joseph's Healthcare and McMaster University, Hamilton, Ontario, Canada. E-mail: searsmamcmaster.ca

ABSTRACT Use of long-acting $\beta$-agonists (LABAs) in asthma remains controversial, and large safety trials are in progress. We have previously reported safety outcomes with formoterol in 117 AstraZeneca asthma trials (78339 patients, 92\% using inhaled corticosteroids) completed by December 2006, and have now added 32 trials with formoterol (26124 patients, 100\% using inhaled corticosteroids) completed by December 2011.

The primary dataset of 79 randomised controlled trials includes 94684 patients, 67380 of whom were exposed to formoterol, while the complete dataset comprises 149 trials and 104463 patients. There were no new asthma-related deaths in the expanded primary dataset, with eight asthma-related deaths among formoterolrandomised patients and two among non-LABA-randomised patients (relative risk 1.13, 95\% CI 0.23-10.9), and 15 versus nine cardiac-related deaths (relative risk $0.47,95 \%$ CI $0.19-1.22$ ). Nonfatal asthma-related serious adverse events were significantly reduced with formoterol (relative risk 0.63, 95\% CI 0.53-0.75), as were discontinuations due to adverse events. Examining 40 trials with direct formoterol versus non-LABA comparisons, Mantel-Haenszel relative risk for asthma-related death was 2.75 (95\% CI 0.52-14.4) and for serious adverse events 0.83 (95\% CI 0.68-1.02).

We conclude that this enlarged dataset indicates no increased risk of asthma-related deaths among patients exposed to formoterol compared with non-LABA treatments, although the wide confidence interval precludes certainty.

@ERSpublications

No increased risk of asthma- or cardiac-related deaths or serious adverse events associated with the use of formoterol http://ow.ly/qfShl

For editorial comments see page 6 .

Received: Jan 092013 | Accepted after revision: May 102013 | First published online: May 302013

Conflict of interest: Disclosures can be found alongside the online version of this article at www.erj.ersjournals.com 


\section{Introduction}

The safety of long-acting $\beta$-agonists (LABAs) in the treatment of asthma has been debated extensively over the past two decades and particularly over the last 6 years since the boxed warning was imposed by the US Food and Drug Administration (FDA) [1]. Concerns initially arose through the results of a clinical trial conducted in the UK with salmeterol versus salbutamol [2] and were heightened by results of a large study in the USA of adding salmeterol or placebo to current therapy [3], in which increased risks of severe asthma outcomes (including mortality) were identified. Many questions were not readily answered because of the structure of those studies, particularly whether the outcomes related directly to adverse effects of LABA treatment or to undertreatment with anti-inflammatory medications such as inhaled corticosteroids (ICS) allowing asthma to be less well controlled despite symptomatic relief from the bronchodilator ("masking”) [4]. Numerous meta-analyses and reviews of clinical trials involving salmeterol and formoterol with and without ICS in patients with asthma have been published [5-13]. Although controversy has continued, there is growing consensus that use of LABAs with adequate ICS is both effective and safe [14, 15].

The FDA recently issued guidelines on asthma management [16]. These included a very strong recommendation against monotherapy with LABAs, restriction of LABA use to those patients in whom control is not achieved with ICS alone, prescription of a combination inhaler containing both LABA and ICS if used in children and adolescents and withdrawal of LABA when asthma control was achieved. These recommendations met with mixed reactions, particularly the recommendation to withdraw LABA therapy when asthma was controlled [17]. Several trials and meta-analyses have subsequently indicated worsening outcomes when following this latter recommendation [18-20].

The FDA has mandated, and pharmaceutical companies have initiated, large clinical trials to determine the safety of LABA by assessing outcomes in patients using LABA with ICS compared with the identical dose of ICS without LABA [21]. These trials will take many years to complete, and even when combined will not have sufficient power to address the primary question of increased risk of asthma mortality [22]. The need for, and relevance of, these large studies has been questioned [23, 24].

In 2009 we published safety outcomes data from all AstraZeneca clinical trials completed up to the end of 2006 involving the use of formoterol in asthma [8]. A detailed analysis of the eight asthma-related deaths in formoterol-randomised patients, with respect to ICS usage, age and other variables, was included in the previous paper and revealed no consistent patterns among any of these variables. In the interests of providing substantial additional data pertinent to recent questions regarding safety of LABAs in asthma, we have updated the earlier analysis by adding a further 32 trials and 26124 patients in recently completed studies.

\section{Methods}

Data source

All AstraZeneca trials in patients with asthma completed by December 2011 involving the use of formoterol either alone as maintenance or reliever therapy or in combination with budesonide were identified through the company database. This involved 104463 patients participating in 149 trials, whereas the earlier report involved 78339 patients participating in 117 trials [8]. The 32 additional trials added 20467 patients randomised to formoterol, 1376 to non-LABA therapy and 4394 to conventional best practice (CBP).

To bring more uniformity into this complete dataset, three groupings of data were constructed, as described below (fig. 1). Since all formoterol-treated patients added to the dataset since the last report also received budesonide, datasets were not split into ICS/non-ICS users, which was one of the major features of the previous paper [8].

\section{Primary dataset}

Because an adverse effect of treatment on asthma severity may require exposure over many months, the main analysis was limited to all randomised, controlled trials with durations of 3-12 months, performed either as centrally run trials or trials run by marketing companies in different countries (locally run). The expanded primary dataset now includes 79 trials, 14 originating from the centrally run AstraZeneca clinical development programmes for formoterol Turbuhaler, 21 from budesonide/formoterol Turbuhaler trials, 14 from budesonide/formoterol pressurised metered-dose inhaler (pMDI) trials, and 30 trials conducted by local AstraZeneca marketing companies with formoterol Turbuhaler or budesonide/formoterol Turbuhaler. The 15 recent trials included in the primary dataset added 17474 formoterol-randomised patients, 642 non-LABA randomised patients and 4394 CBP-randomised patients. In total, these 79 trials include 94684 patients, of whom 67380 were randomised to formoterol-containing products, 4170 to salmeterol-containing products, 4394 to CBP and 18740 to non-LABA products. The results for salmeterol-containing products and CBP have 


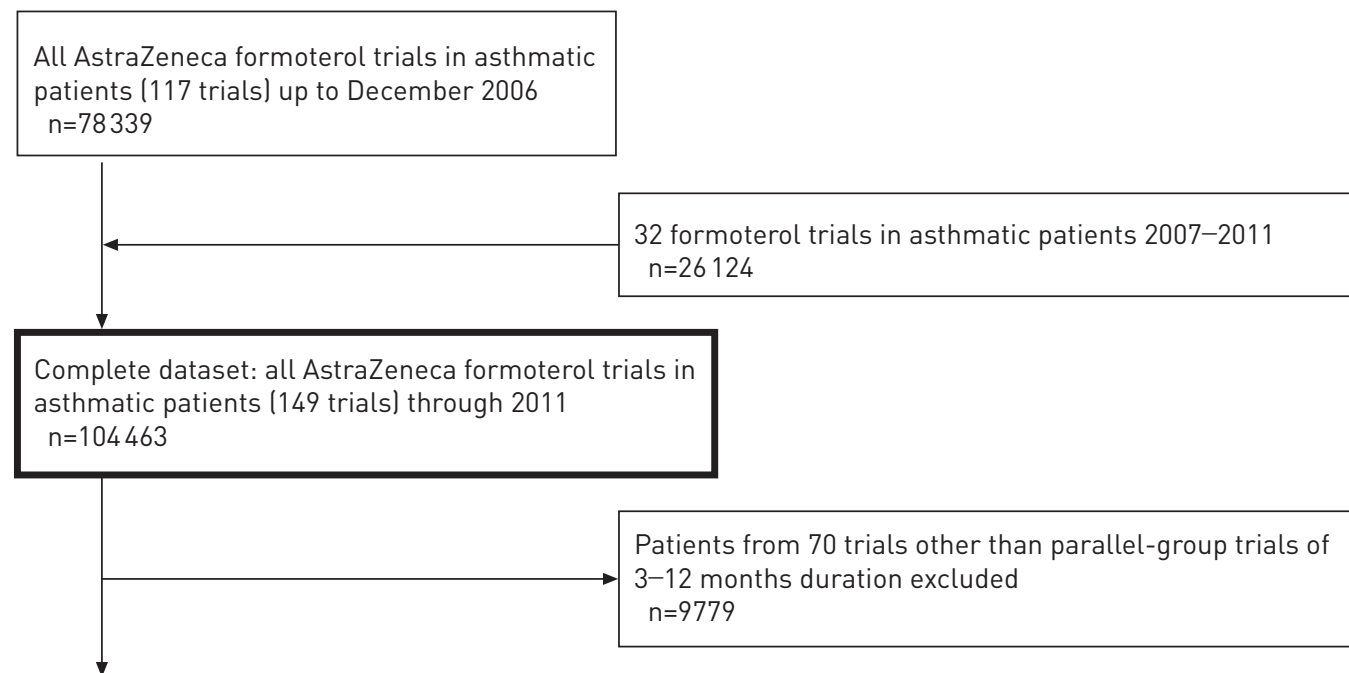

All AstraZeneca formoterol parallel-group trials of 3-12 months duration (79 trials)

$\mathrm{n}=94684$

$\begin{array}{ll}\text { FORM } & \mathrm{n}=67380 \\ \text { Non-LABA } & \mathrm{n}=18740 \\ \text { CBP-randomised patients } & \mathrm{n}=4394 \\ \text { Salmeterol-randomised patients } & \mathrm{n}=4170\end{array}$

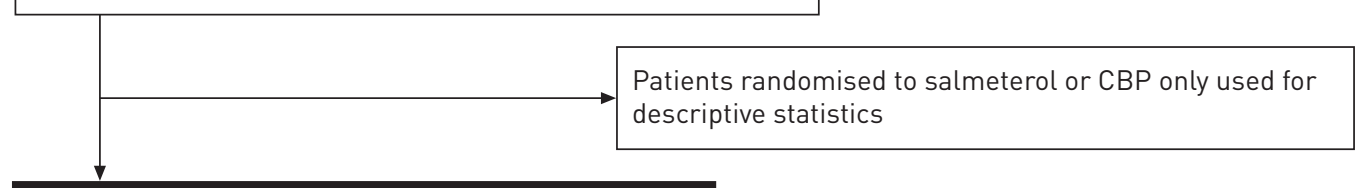

Primary dataset for statistical analysis (79 trials):

$\begin{array}{ll}\text { FORM } & \mathrm{n}=67380 \\ \text { Non-LABA } & \mathrm{n}=18740\end{array}$

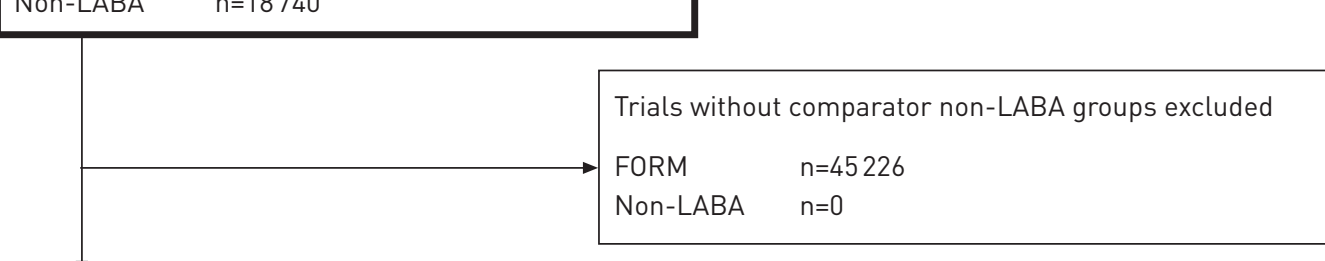

Mantel-Haenszel dataset (40 trials comparing

FORM with non-LABA):

FORM $\quad \mathrm{n}=22154$

Non-LABA $\quad n=18740$

FIGURE 1 Flow chart of all patients involved in AstraZeneca trials with formoterol in asthmatic patients. FORM: formoterol randomised; non-LABA: non-long acting $\beta$-agonist-randomised; CBP: conventional best practice.

been included for completeness, but were not subjected to statistical analyses, as the comparison of interest in the primary dataset is between formoterol-containing products and non-LABA products.

\section{Complete dataset for supplementary analysis}

The complete dataset includes all identified AstraZeneca asthma trials, regardless of duration and study design, to ensure that no important safety signals were missed by selecting only trials of 3-12 months duration. These included all centrally run trials in asthmatic patients, including long-term safety studies, emergency room trials in acute severe asthma, pharmacokinetic and high-dose tolerance studies, methacholine-induced bronchoconstriction studies and trials in a prematurely terminated (due to device malfunction) formoterol pMDI programme. All identified locally run parallel-group trials in asthmatic 
patients were also included. Results from this supplementary analysis are summarised in this paper and are further described in the online supplementary material.

\section{Dataset for Mantel-Haenszel analysis}

Trials of 3-12 months duration which involved a direct comparison between formoterol treatments and non-LABA treatments $(\mathrm{n}=40)$ were combined for calculation of exposure-adjusted Mantel-Haenszel relative risks.

\section{Outcome events}

All-cause mortality, asthma-related deaths and nonfatal asthma-related serious adverse events (SAEs) were co-primary end-points. Secondary outcomes were overall SAEs, nonfatal cardiac-related SAEs, discontinuations due to adverse events (DAEs), and asthma-related and cardiac-related DAEs. Analysis of asthma-related SAEs by sex, age and race was performed using descriptive statistics. All these events were evaluated at the time by the investigators involved in each study and prior to unblinding. All fatalities in all trials were reassessed by the present authors, and categorised as asthma-related, cardiac-related or due to other reasons.

Asthma-related events were defined as any event coded to the preferred terms "asthma", "status asthmaticus", "asthmatic crisis" or "bronchospasm" according to the Medical Dictionary for Regulatory Activities (MedDRA) version 15.0 (McClean, VA, USA). In addition, one death in the trial by KunA et al. [25] and one death in the trial by VON BERG et al. [26] reported by the trial investigators as due to respiratory failure, were considered by the current authors as asthma-related. Cardiac-related events were defined as any event coded using MedDRA version 15.0 according to the terms in online supplementary table S1.

SAEs (both asthma-related and cardiac-related) were defined using the FDA International Conference on Harmonisation recommendations, i.e. any adverse event that was immediately life threatening, required inpatient hospitalisation or prolongation of existing hospitalisation, resulted in persistent or significant disability or incapacity, was a congenital abnormality/birth defect or was an important medical event that may jeopardise the subject or require medical intervention to prevent one of the outcomes listed above.

Events were counted as the number of patients reporting at least one such event. When multiple events were recorded, a patient was counted once for each group of adverse events. Thus, a patient reporting both angina pectoris and hypertension would be counted once in the group of cardiac-related events, while a patient reporting two asthma attacks and one angina pectoris would be counted once among the asthmarelated events and once among the cardiac-related events.

Deaths and discontinuations of study treatment due to adverse events were represented by the event resulting in the death or discontinuation. For SAEs and adverse events, multiple occurrences of the same event were counted as the first incidence of the event. Events with the outcome of death are counted among DAEs but not among nonfatal SAEs.

\section{Data analyses}

Primary dataset

For each patient, the person-time of follow-up in the trial was measured and cumulated to obtain personyears of exposure. The rate of fatal outcome events was expressed per 1000 person-years computed for each treatment group (1000 treatment-years (TTY)). The crude rate ratio associated with formoterol use and its confidence interval were computed by the exact method (StatXact version 8.0.0; Cytel Inc., Cambridge, MA, USA) [27]. For nonfatal events, the crude rate ratio was approximated by the odds ratio obtained from StatXact using the number of randomised patients and the number of patients experiencing at least one event.

\section{Complete dataset}

The complete dataset was subjected to descriptive statistics only.

\section{Mantel-Haenszel dataset}

Overall relative risks were analysed using a stratified Mantel-Haenszel approach adjusted for treatment exposure, which allowed for possible differences between trials, thereby reducing bias. This provided the pooled Mantel-Haenszel relative risk and 95\% confidence intervals for each outcome event. For relative risks, differences were considered statistically significant when the 95\% confidence intervals excluded 1.00.

Formoterol doses are expressed as delivered doses. Formoterol delivered doses of $9 \mu \mathrm{g}$ and $18 \mu \mathrm{g}$ correspond to metered doses of $12 \mu \mathrm{g}$ and $24 \mu \mathrm{g}$, respectively. 
The analyses undertaken by the FDA [5] excluded products not approved for clinical use in the USA. The formoterol-containing product produced by AstraZeneca that is approved in the USA is budesonide/ formoterol pMDI. This paper also utilises data related to formoterol Turbuhaler and budesonide/ formoterol Turbuhaler, which have approvals in $>100$ countries worldwide, but not in the USA.

It is important to note that although our comparison comes from a dataset of randomised controlled trials this does not mean that the estimates of safety in the primary analysis come from randomised comparisons. Many trials randomised participants to different doses of budesonide/formoterol but with no other comparator, and when these trials are compared in the dataset to other trials, the results could potentially be confounded by differences in entry criteria, severity, level of monitoring and other differences between trials. However, the smaller Mantel--Haenszel analysis is protected by randomisation to formoterol or nonLABA comparator.

\section{Results}

\section{Patients and events}

Online supplementary table S2 presents separately the numbers of patients and deaths in the data previously reported and in the additional recent trials. Details of the recent trials are presented in online supplementary tables S3 and S4, and deaths and adverse events in these trials in online supplementary tables S5-S9.

\section{Primary dataset \\ Number of patients and total exposure}

The primary dataset included 79 trials of 3-12 months duration. The 15 additional trials added 17474 formoterol-randomised patients, 642 non-LABA-randomised patients and 4394 CBP-randomised patients to the primary dataset. The number of patients per treatment group and the total exposure time per treatment group (expressed in TTY) are summarised in table 1. Randomisation had allocated 67380 patients to formoterol-containing products (exposure 33.7 TTY) and 18740 to non-LABA products (exposure 9.5 TTY).

\section{Deaths}

In total, 71 patients died during trials included in the primary dataset (table 2). Of these, 50 were randomised to formoterol, 15 to non-LABA treatments, three to salmeterol and three to CBP. All-cause mortality was similar between patients randomised to formoterol and non-LABA treatments (1.5 and 1.6 deaths per TTY, respectively; rate ratio 0.94, 95\% CI 0.52-1.80) (tables 3 and 4). 10 deaths were considered asthma-related; eight among formoterol-randomised patients and two among non-LABA-randomised patients ( 0.24 and 2.1 deaths per TTY, respectively; rate ratio $1.13,95 \%$ CI $0.23-10.9)$. Cardiac-related deaths were reported less frequently on LABA (rate ratio $0.47,95 \%$ CI $0.19-1.22$ ), while deaths from causes other than asthma or cardiac events were reported more frequently on formoterol (rate ratio 1.90, 95\% CI 0.66-7.48). There were no asthma-related deaths reported from the recent trials.

\section{SAES}

Nonfatal asthma-related SAEs were significantly reduced with formoterol compared to non-LABA treatment (rate ratio 0.63 , 95\% CI: 0.53-0.75) (table 3 and fig. 2). There was no evidence of increased risk of nonfatal asthma-related SAEs associated with formoterol in any subgroup of patients by age, sex or ethnicity (table 4). Specifically, no increased risk for asthma-related SAEs was observed in black patients or

TABLE 1 Number of patients and total exposure (1000 treatment years (TTY)), by randomised treatment in the primary, complete and Mantel-Haenszel datasets

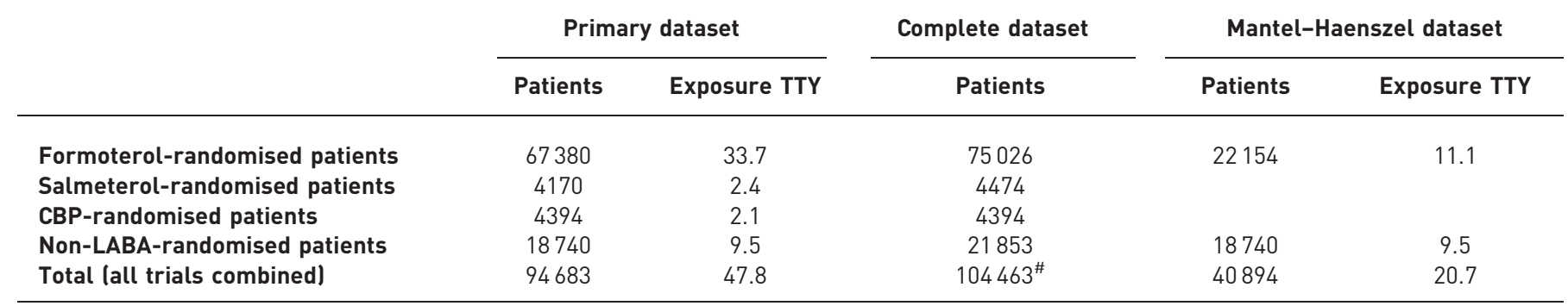

Data are presented as $n$. Patients in crossover trials have only been counted once in this total. Note that $n=105746$ in the four treatment groups. CBP: current best practice; LABA: long-acting $\beta$-agonist. " : unique patients. 

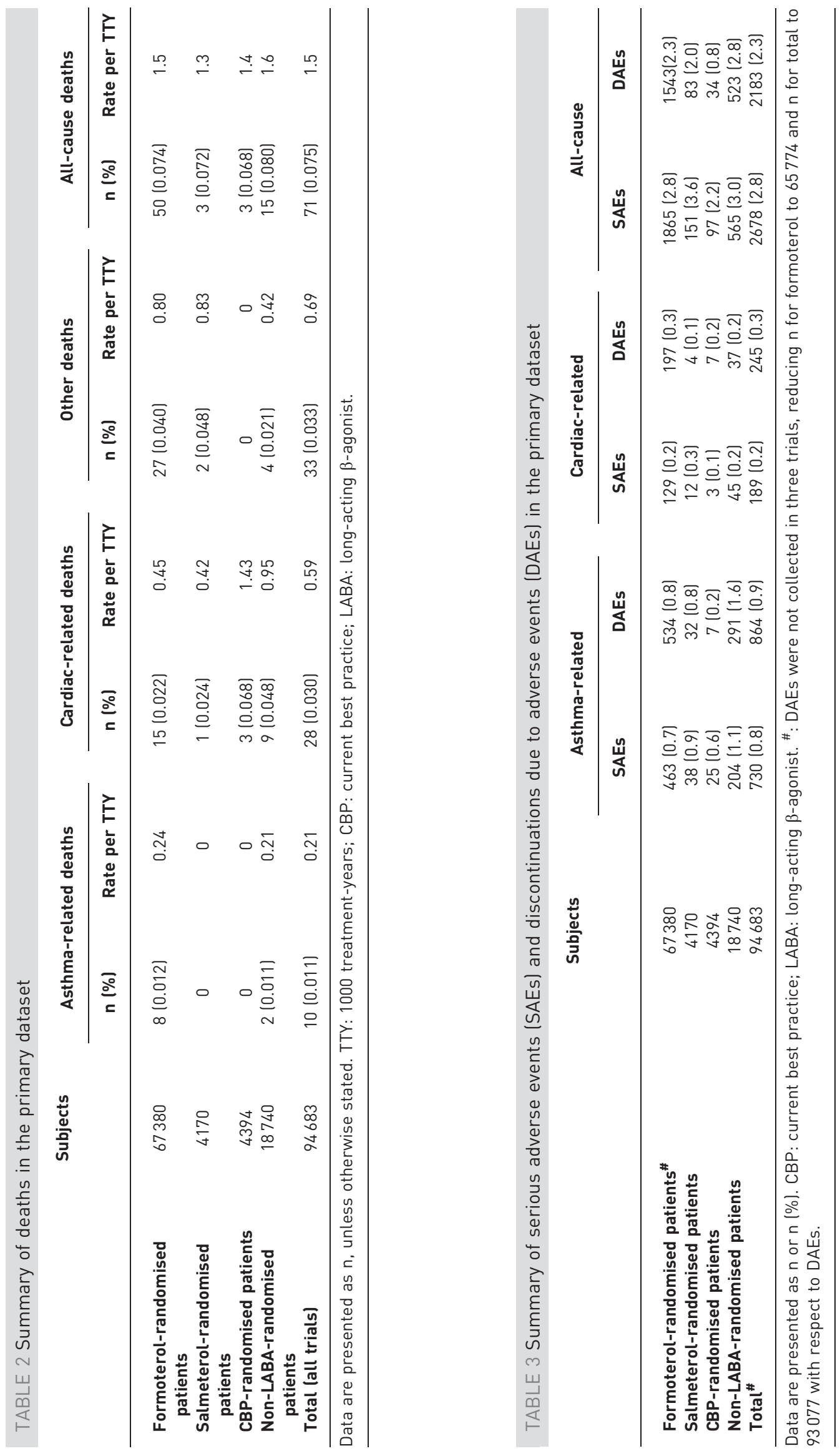
TABLE 4 Numbers of patients reporting at least one asthma-related nonfatal serious adverse event by age, sex and race in the primary dataset

Formoterol-containing products

Non-LABA-containing products

\begin{tabular}{lcc}
\hline Age group years & & \\
$4-11$ & $39 / 3264(1.2)$ & $25 / 2165(1.2)$ \\
$12-17$ & $24 / 5088(0.5)$ & $17 / 1989(0.9)$ \\
$18-64$ & $335 / 52492(0.6)$ & $141 / 13911(1.1)$ \\
$>65$ & $65 / 6494(1.0)$ & $21 / 1467(1.4)$ \\
Unknown & $0 / 42(0.0)$ & $0 / 0(0.0)$ \\
Sex & & \\
Male & $174 / 28627(0.7)$ & $72 / 8264(0.9)$ \\
Female & $289 / 38577(0.8)$ & $132 / 10353(1.3)$ \\
Unknown & $0 / 176(0.0)$ & $0 / 123(0.0)$ \\
Race & & $123 / 14931(0.8)$ \\
Caucasian & $288 / 44581(0.6)$ & $8 / 847(0.9)$ \\
Black & $11 / 1434(0.8)$ & $53 / 1916(2.8)$ \\
Oriental & $103 / 5398(1.9)$ & $20 / 1046(1.9)$ \\
Other & $25 / 2335(1.1)$ & $0 / 0(0.0)$ \\
Unknown & $36 / 16362(0.3)$ & $204 / 18740(1.1)$ \\
Total & $463 / 67380(0.7)$ & \\
\hline
\end{tabular}

Data are presented as $\mathrm{n} / \mathrm{N}(\%)$. LABA: long-acting $\beta$-agonist.

in children and adolescents. There was no increased risk of nonfatal asthma-related SAEs related to increased doses of formoterol by randomised treatment (table 5).

No increased risk was observed for formoterol versus non-LABA treatments for nonfatal cardiac-related SAEs (rate ratio $0.79,95 \%$ CI $0.53-1.15$ ) or all-cause SAEs (rate ratio 0.91, 95\% CI 0.83-1.01) (table 3 and fig. 2).

All-cause death
Asthma-related death
Cardiac-related death
Other death
All-cause SAE
Asthma-related SAE
Cardiac-related SAE
All-cause DAE
Asthma-related DAE
Cardiac-related DAE

All-cause death

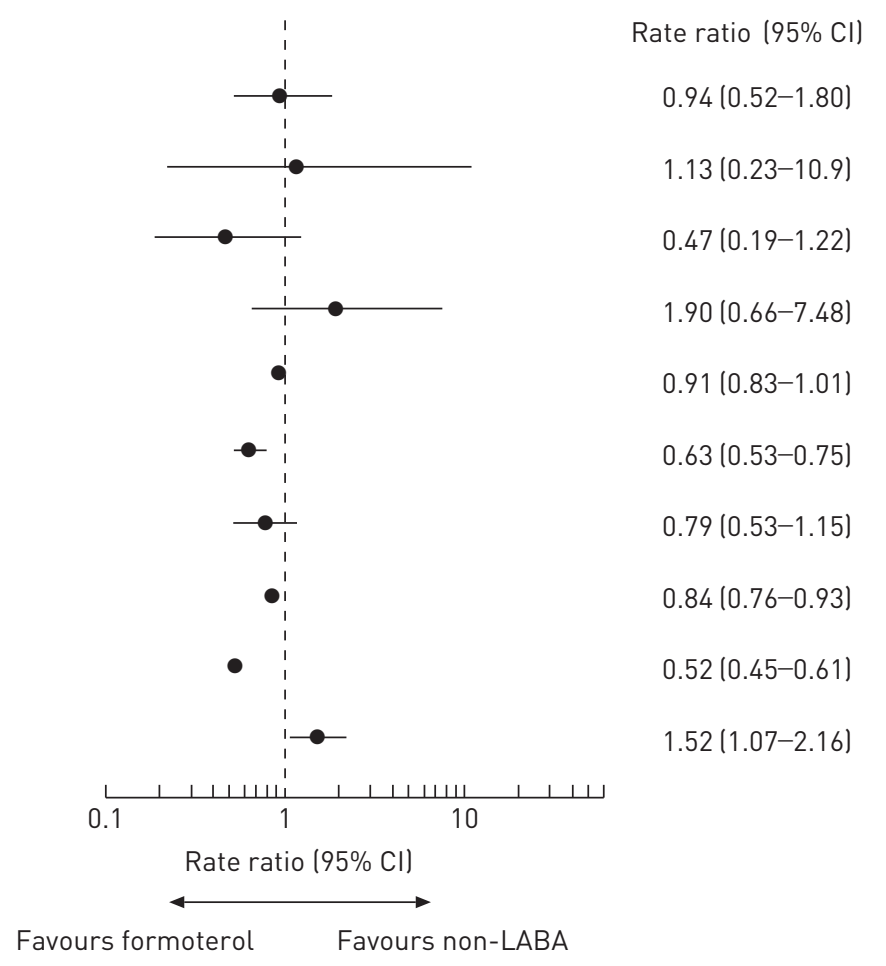

FIGURE 2 Combined results for formoterol versus non-long-acting $\beta$-agonist (LABA) treatment from the primary dataset. SAE: serious adverse event; DAE: discontinuation due to adverse event. 
TABLE 5 Number of asthma-related serious adverse events (SAEs) by daily dose of formoterol in the primary dataset

$\begin{array}{lcr}9 \boldsymbol{\mu} \mathbf{g}^{\#} & 6348 & 88(1.39) \\ \mathbf{1 8} \boldsymbol{\mu g} & 16643 & 95(0.57) \\ \mathbf{3 6} \boldsymbol{\mu g} & 909 & 4(0.44) \\ \text { As-needed use, adjustable dosing or uncertain dose } & 43480 & 276(0.63) \\ \text { Total } & 67380 & 463(0.79)\end{array}$

Data are presented as $\mathrm{n}$ or $\mathrm{n}(\%) .{ }^{*}$ : includes the 114 children who received $80 / 4.5 \mu \mathrm{g}$ budesonide/formoterol + terbutaline as needed in trial SD039-0673.

DAES

All-cause DAEs (rate ratio 0.84, 95\% CI 0.76-0.93) and asthma-related DAEs (rate ratio 0.52, 95\% CI 0.45-0.61) were significantly reduced with formoterol compared to non-LABAs (table 3 and fig. 2). For cardiac-related DAEs a statistically significant increase was observed (rate ratio 1.52, 95\% CI 1.07-2.16).

\section{Complete dataset for supplementary analysis}

Numbers of trials and patients

The supplementary analyses, which included all available trials in asthmatic patients irrespective of duration and design (see the methods section), added 70 trials to those in the primary analyses, giving 149 trials and 104463 patients (table 1). Of these, 75026 were randomised to formoterol and 21853 to a non-LABA regimen. The 32 recent trials added 20467 randomised to formoterol, 1376 to non-LABA treatments and 4394 to CBP to the complete dataset. Patients in crossover trials have been counted once for each exposed treatment, but only once in the totals.

Asthma-related, cardiac-related and all-cause mortality

The supplementary analyses added six deaths to those in the primary analyses. Of the 77 deaths, 11 were asthma-related and 31 cardiac-related (table 6). There was one additional asthma-related death in the 17 recent trials included in the supplementary dataset but not in the primary dataset; this occurred in an uncontrolled trial in which all patients received budesonide/formoterol maintenance and reliever therapy.

All-cause mortality across all trials in the supplementary dataset was $0.07 \%$ for formoterol-randomised patients, $0.07 \%$ for non-LABA-randomised patients, $0.07 \%$ for CBP-randomised patients and $0.07 \%$ for salmeterol-randomised patients. These results do not differ from those of the primary analysis.

Asthma-related and cardiac-related SAES

Across all trials in the supplementary analyses, 495 (0.7\%) formoterol-randomised patients reported at least one nonfatal asthma-related SAE and $141(0.2 \%)$ patients reported at least one nonfatal cardiac-related SAE. The corresponding numbers among the non-LABA-randomised patients were $213(1.0 \%)$ and $47(0.2 \%)$ (table 6). These results do not differ from those of the primary analysis.

\section{TABLE 6 Summary of results from the complete dataset}

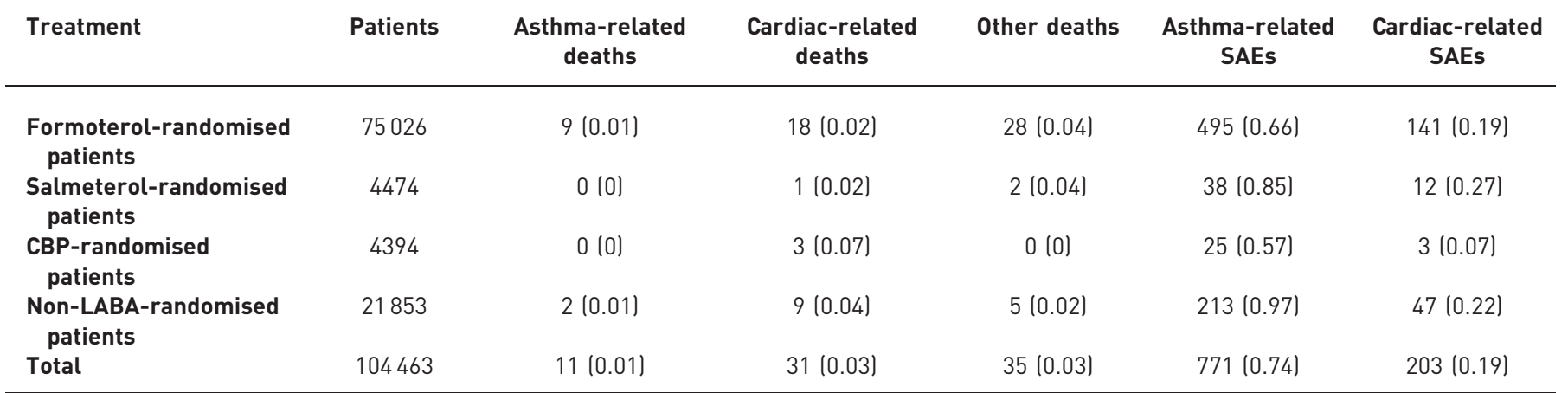

Data are presented as $n$ or $n(\%)$. SAE: serious adverse event; CBP: current best practice; LABA: long-acting $\beta$-agonist. 
Mantel-Haenszel dataset

For the 40 trials in the primary dataset that included a direct formoterol versus non-LABA comparison, the overall relative risk was calculated using a Mantel-Haenszel approach, stratified by study and adjusted for treatment exposure (fig. 3). This dataset included 22154 patients randomised to formoterol (11.1 TTY) and 18740 randomised to non-LABA treatments (9.6 TTY). The Mantel-Haenszel relative risk for asthma death was 2.75 (95\% CI 0.52-14.4), while for asthma-related SAEs the relative risk was close to statistical significance in favour of formoterol treatment $(0.83,95 \%$ CI $0.68-1.02)$. It should be noted that since an asthma-related death only occurred in four out of the 40 trials (six deaths on formoterol and two on nonLABA treatment), data from 36 trials were not used in the analysis. For the other safety parameters, the results were similar to those from the full primary dataset. Detailed results are presented in online supplementary table S10.

\section{Discussion}

This report adds to the primary dataset the outcomes from a further 17474 patients exposed to formoterol (10.1 TTY) in trials completed or reported since our 2009 publication [8]. There were no asthma-related deaths among these 17474 patients, even though this number is larger than the number of patients exposed to either salmeterol or placebo in the Salmeterol Multicentre Asthma Research trial [3]. Of note, all patients using formoterol in the recent data also used ICS, which was not the case in the Serevent Nationwide Surveillance Study [2] or the Salmeterol Multicenter Asthma Research Trial [3].

The likely relevance of use of ICS when LABAs are prescribed was identified by MCMAHON et al. [28], who examined the effect of age on the safety outcomes assessed in the meta-analysis conducted for the FDA by LEVENSON [5] and presented at the Joint Advisory Committee hearing in 2008. The risk of an adverse outcome (using the composite outcome of asthma-related death, intubation or hospitalisation) was highest in children and adolescents and was not substantially altered by "concomitant ICS use" (i.e. any ICS use including use recorded at baseline, but information on the frequency of usage, if any, throughout the trial was not available). However such risk was not evident with "assigned ICS use" (the patients were prescribed ICS on a regular scheduled regimen as part of the trial design, either in a combination LABA/ICS inhaler or a separate ICS device). The absence of risk associated with LABA with mandatory use of ICS was in fact reported by LEVENSON [5] in his original report.

All-cause death
Asthma-related death
Cardiac-related death
Other death
All-cause SAE
Asthma-related SAE
Cardiac-related SAE
All-cause DAE
Asthma-related DAE
Cardiac-related DAE

All-cause death

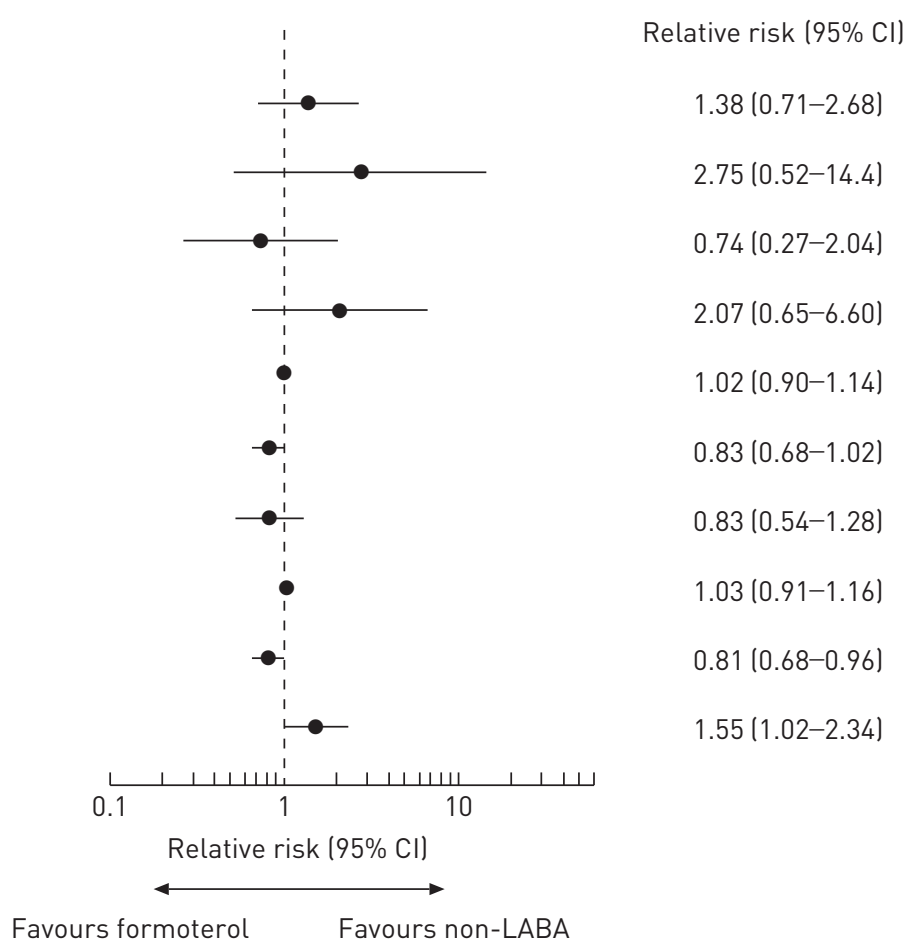

FIGURE 3 Combined results for formoterol versus non-long-acting $\beta$-agonist (LABA) treatment for the primary dataset for a subset of 40 formoterol versus non-LABA trials (Mantel-Haenszel dataset). SAE: serious adverse event; DAE: discontinuation due to adverse event. 
The relative risk of mortality in the expanded primary dataset of 1.13 (95\% CI $0.23-10.19$ ) indicates no increased risk for asthma-related deaths among patients exposed to formoterol compared with non-LABA treatments. Further, formoterol treatment was associated with significant reductions in asthma-related SAEs (mainly hospitalisations) with a relative risk of 0.63 (95\% CI 0.53-0.75). No increased risks for cardiac or all-cause mortality or cardiac-related SAEs were observed. However, the limitations noted in the previous paper persist, namely that mortality is a rare event in these trials and estimates of risk must be interpreted with caution given that the trials were not powered on these events. As there were no asthma-related deaths in the additional studies of the primary dataset, the absolute risk of death for study participants overall is low. However, due to the low number of events available for analysis, the $95 \%$ confidence interval around the relative risk remains wide (0.23-10.9), precluding absolute certainty about the results.

Other limitations of the study include the inhomogeneity of the primary dataset as described in our previous paper [8], although this has been attenuated with the inclusion of a large number of trials that did not have a non-LABA comparator arm. The approach we have taken is the only possible way to utilise data from all trials with formoterol-containing products when looking for rare events such as asthma-related mortality. The more stringent approach, the Mantel-Haenszel analysis of all 40 trials that had a formoterol and a non-LABA treatment arm, gave a relative risk for asthma death of 2.75 (95\% CI 0.52-14.4); however, this used data from only four trials. For asthma-related SAEs, this approach gave a relative risk of $0.83(95 \%$ CI 0.68-1.02). It should be noted that either approach gives a relative risk for asthma-related SAE that favours treatment with formoterol, and similar relative risks for all of the safety parameters, with the exception of asthma death, as illustrated in figures 2 and 3.

Concern has been expressed regarding racial susceptibility to adverse effects of LABAs. The primary dataset now includes 1434 black patients for whom no increased risk of asthma-related SAEs was detected. Three recent publications have likewise failed to show increased risks in black patients using LABAs with mandatory ICS. SPECTOR et al. [29] treated 311 black patients with budesonide/formoterol versus budesonide, and reported increased benefit in lung function and asthma control with similar safety profiles. BROWN et al. [30] treated 741 black patients for 1 year with budesonide/formoterol versus budesonide, and found a decreased exacerbation rate with budesonide/formoterol and a similar safety profile between the two treatments. In a real-life setting, Wells et al. [31] reviewed data from a large health maintenance organisation, including 668 African-Americans, and reported better outcomes (fewer severe exacerbations) with ICS/LABA (dominantly fluticasone/salmeterol) compared with ICS alone, with no safety issues identified.

Concern has also been expressed regarding the lack of clear evidence of safety of the use of LABAs in the management of children with asthma. Our data do suggest that the benefit of formoterol is reduced in children compared with adults, but with no evidence of any increase in events (table 4). Event rates are similar between adults and children in the non-LABA control arms and similar rates were seen in children in the formoterol arms. However, clear improvements can be seen in the adolescent and adult arms with formoterol-containing products. The improvement in adolescents is important as this age group may be less adherent to treatment, especially with multi-inhaler treatment regimens.

We recognise that, even with the addition of 32 recent trials involving formoterol, of which 15 contributed to the primary analysis, the outcomes are still less precise than those anticipated to result from the FDAmandated megatrials which will involve some 50000 patients, half of whom will be exposed to LABA with ICS and half to the same dose of ICS without LABA [21]. However, the data reported in the present paper, together with the many other meta-analyses and systematic reviews of the existing literature that have been undertaken [5-13], are consistent with the likelihood that the problem associated with use of LABAs in asthma is not an intrinsic safety issue related to the LABA itself, but to the overall management of the condition and the lack of appropriate treatment with ICS [23, 28, 32]. A number of Cochrane reviews [33-37] have addressed the safety of LABA/ICS combinations compared to use of ICS alone, and concluded that while combination therapy in general is favourable compared to ICS-alone treatment in adults, the results are less clear in children for whom limited data are yet available. The FDA trials, which appropriately mandate that all patients are treated with an ICS, are markedly underpowered for any outcome related to mortality, but are well powered to examine SAEs related to asthma as well as other SAEs [22]. The inclusion of specified numbers of children is one of the strengths of the trials mandated by the FDA, since data on asthma-related SAEs are sparse for ICS versus LABA-ICS combinations in young patients.

In conclusion, we provide evidence that the use of formoterol in asthma patients, most of whom used ICS, is not associated with an increased risk of asthma-related or cardiac-related deaths or SAEs. In the recent trials, in which all patients used ICS, no asthma-related deaths were seen in the primary analysis, while asthmarelated SAEs were reduced. No increased risks for asthma-related events were observed in the black or any other racial subpopulation. We report these new data to reassure physicians and patients that formoterol used appropriately with mandatory ICS is effective and safe. This is particularly relevant given the increasing 
evidence that withdrawal of LABA from patients who have achieved better control on combination therapy results in loss of asthma control compared with continued combined LABA/ICS treatment.

\section{References}

1 Murphy S, Roberts R. "Black-box" 101: how the Food and Drug Administration evaluates, communicates, and manages drug benefit/risk. J Allergy Clin Immunol 2006; 117: 34-39.

2 Castle W, Fuller R, Hall J, et al. Serevent nationwide surveillance study: comparison of salmeterol with salbutamol in asthmatic patients who require regular bronchodilator treatment. BMJ 1993; 306: 1034-1037.

3 Nelson HS, Weiss ST, Bleecker ER, et al. The Salmeterol Multicenter Asthma Research Trial: a comparison of usual pharmacotherapy for asthma or usual pharmacotherapy plus salmeterol. Chest 2006; 129: 15-26.

4 McIvor RA, Pizzichini E, Turner MO, et al. Potential masking effects of salmeterol on airway inflammation in asthma. Am J Respir Crit Care Med 1998; 158: 924-930.

5 M. Levenson. Long-acting Beta-agonists and Adverse Asthma Events Meta-analysis. Statistical briefing package for joint meeting of the Pulmonary-Allergy Drugs Advisory Committee, Drug Safety and Risk Management Advisory Committee and Paediatric Advisory Committee, 2008. www.fda.gov/ohrms/dockets/ac/cder08.html\# PulmonaryAllergy Date last updated: November 12, 2008. Date last accessed: November 6, 2013.

6 Bateman E, Nelson H, Bousquet J, et al. Meta-analysis: effects of adding salmeterol to inhaled corticosteroids on serious asthma-related events. Ann Intern Med 2008; 149: 33-42.

7 Jaeschke R, O'Byrne PM, Mezja F, et al. The safety of long-acting $\beta$-agonists among patients with asthma using inhaled corticosteroids. Systematic review and meta-analysis. Am J Respir Crit Care Med 2008; 178: 1009-1016.

8 Sears MR, Ottosson A, Radner F, et al. Long-acting beta-agonists: a review of formoterol safety data from asthma clinical trials. Eur Respir J 2009; 33: 21-32.

9 Rodrigo GJ, Moral VP, Marcos LG, et al. Safety of regular use of long-acting beta agonists as monotherapy or added to inhaled corticosteroids in asthma. A systematic review. Pulm Pharmacol Ther 2009; 22: 9-19.

10 Cates CJ, Lasserson TJ, Jaeschke R. Regular treatment with salmeterol and inhaled steroids for chronic asthma: serious adverse events. Cochrane Database Syst Rev 2009; 3: CD006922.

11 Sears MR, Radner F. Safety of budesonide/formoterol maintenance and reliever therapy in asthma trials. Respir Med 2009; 103: 1960-1968.

12 Nelson H, Bonuccelli C, Radner F, et al. Safety of formoterol in patients with asthma: combined analysis of data from double-blind, randomized controlled trials. J Allergy Clin Immunol 2010; 125: 390-396.

13 Price JF, Radner F, Lenney W, et al. Safety of formoterol in children and adolescents: experience from asthma clinical trials. Arch Dis Child 2010; 95: 1047-1053.

14 Nelson HS, Carr W, Nathan R, et al. Update on the safety of long-acting beta-agonists in combination with inhaled corticosteroid for the treatment of asthma. Ann Asthma Allergy Immunol 2009; 102: 11-15.

15 Rodrigo GJ, Castro-Rodríguez JA. Safety of long-acting $\beta$ agonists for the treatment of asthma: clearing the air. Thorax 2012; 67: 342-349.

16 Chowdhury BA, Dal Pan G. The FDA and safe use of long-acting beta-agonists in the treatment of asthma. $N$ Engl $J$ Med 2010; 362: 1169-1171.

17 Lemanske RF, Busse WW. The US Food and Drug Administration and long-acting $\beta_{2}$-agonists: the importance of striking the right balance between risks and benefits of therapy? J Allergy Clin Immunol 2010; 126: 449-452.

18 Reddel HK, Gibson PG, Peters MJ, et al. Down-titration from high-dose combination therapy in asthma: removal of long-acting $\beta_{2}$-agonist. Respir Med 2010; 104: 1110-1120.

19 Hagiwara M, Delea TE, Stanford RH, et al. Stepping down to fluticasone propionate or a lower dose of fluticasone propionate/salmeterol combination in asthma patients recently initiating combination therapy. Allergy Asthma Proc 2010; 31: 203-210.

20 Brozek JL, Kraft M, Krishnan JA, et al. Long-acting $\beta_{2}$-agonist step-off in patients with controlled asthma. Arch Intern Med 2012; 172: 1365-1375.

21 Chowdhury BA, Seymour SM, Levenson MS. Assessing the safety of adding LABAs to inhaled corticosteroids for treating asthma. N Engl J Med 2011; 364: 2473-2475.

22 Sears MR. Safety of long-acting beta-agonists: are new data really required? Chest 2009; 136: 604-607.

23 Szefler SJ, Busse WW, for the Paediatric and Joint Adult Steering Committees. Adding LABAs to inhaled glucocorticoids for asthma. N Engl J Med 2011; 365: 1260-1261.

24 Sears MR. The FDA-mandated trial of safety of long-acting beta-agonists in asthma: finality or futility? Thorax 2013; 68: 195-198.

25 Kuna P, Peters MJ, Manjra AI, et al. Effect of budesonide/formoterol maintenance and reliever therapy on asthma exacerbations. Int J Clin Pract 2007; 61: 725-736.

26 Von Berg A, Papageorgiou Saxoni F, Wille S, et al. Efficacy and tolerability of formoterol Turbuhaler in children. Int J Clin Pract 2003; 57: 852-856.

27 StatXact. www.cytel.com/software/statxact Date last accessed: November 7, 2013.

28 McMahon AW, Levenson MS, McEvoy BW, et al. Age and risks of FDA-approved long-acting $\beta_{2}$-adrenergic receptor agonists. Pediatrics 2011; 128: e1147-e1154.

29 Spector SL, Martin UJ, Uryniak T, et al. Budesonide/formoterol pressurized metered-dose inhaler versus budesonide: a randomized controlled trial in black patients with asthma. J Asthma 2012; 49: 70-77.

30 Brown RW, O’Brien CD, Martin UJ, et al. Long-term safety and asthma control measures with a budesonide/ formoterol pressurized metered-dose inhaler in African American asthmatic patients: a randomized controlled trial. J Allergy Clin Immunol 2012; 130: 362-367.

31 Wells KE, Peterson EL, Ahmedani BK, et al. The relationship between combination inhaled corticosteroid and longacting $\beta$-agonist use and severe asthma exacerbations in a diverse population. J Allergy Clin Immunol 2012; 179: $1274-1279$.

32 Sears MR. Safe use of long-acting $\beta$-agonists: what have we learnt? Expert Opin Drug Saf 2011; 10: 767-778.

33 Cates CJ, Oleszczuk M, Stovold E, et al. Safety of regular formoterol or salmeterol in children with asthma: an overview of Cochrane reviews. Cochrane Database Syst Rev 2012; 10: CD010005. 
34 Ni Chroinin M, Greenstone I, Lasserson TJ, et al. Addition of long-acting beta2-agonists to inhaled steroids as first line therapy for persistent asthma in steroid-naive adults and children. Cochrane Database Syst Rev 2009; 4 : CD005307.

35 Ni Chroinin M, Lasserson TJ, Greenstone I, et al. Addition of long-acting beta-agonists to inhaled corticosteroids for chronic asthma in children. Cochrane Database Syst Rev 2009; 3: CD007949.

36 Ducharme FM, Ni Chroinin M, Greenstone I, et al. Addition of long-acting beta2-agonists to inhaled steroids versus higher dose inhaled steroids in adults and children with persistent asthma. Cochrane Database Syst Rev 2010; 4: CD005533.

37 Ducharme FM, Ni Chroinin M, Greenstone I, et al. Addition of long-acting beta2-agonists to inhaled corticosteroids versus same dose inhaled corticosteroids for chronic asthma in adults and children. Cochrane Database Sys Rev 2010; 5: CD005535. 